\title{
Familienleistungen bei grenzüberschreitenden Sachverhalten
}

\author{
Renata Babińska-Górecka und Karolina Stopka
}

A. Einführung 285

B. Begriff und Koordinierung der Familienleistungen 287

I. Begriff der Familienleistungen nach polnischem Recht 287

II. Anwendbarkeit bei grenzüberschreitenden Sachverhalten 289

III. Zur Frage der Kumulierung 291

IV. Begriff der Familienleistungen nach Unionsrecht 293

V. Folgerungen für die Qualifizierung nach polnischem Recht 294

C. Weitere Problemstellungen 297

\section{A. Einführung}

Familienleistungen wurden in den Gesetzgebungen aller EU-Mitgliedsstaaten vorgesehen und gehören zu den häufigsten Leistungen, die von den Angestellten innerhalb der Union und dem Europäischen Wirtschaftsraum in Anspruch genommen werden. ${ }^{1}$ Außerdem beeinflusst die Erwerbsmigration eines Familienmitgliedes auf der Ebene der Familienleistungen - im Gegensatz zu anderen, von der Koordinierung der Sozialversicherungssysteme umfassten Leistungen - unmittelbar den Rechteumfang anderer Familienmitglieder. Demzufolge gewinnt die Regelung von Koordinierungsgrundsätzen derartiger Leistungen eine besondere Bedeutung. ${ }^{2}$ Die Entscheidung über Koordinierungsangelegenheiten in Bezug auf die Familienleistungen erfolgt durch Anwendung einer Reihe rechtlicher Regelungen, die in der Verordnung Nr. 883/2004³ sowie in nationalen

1 G. Uścińska, Koordynacja świadczeń rodzinnych w regulacjach wspólnotowych, in: Polityka Społeczna Nr. 8 2007, S. 45; M. Rusewicz, Zasady nabywania świadczeń rodzinnych w Unii Europejskiej, in: Prawo i Podatki Unii Europejskiej Nr. 5 2007, S. 7.

2 G. Uścińska, Konsekwencje integracji europejskiej dla ubezpieczeń społecznych, in: Ewolucja ubezpieczeń społecznych w okresie transformacji ustrojowej, Bydgoszcz 2011, S. 41-42; L. Mitrus, Zakres przedmiotowy koordynacji systemów zabezpieczenia społecznego w Unii Europejskiej, in: Z zagadnień prawa pracy i prawa socjalnego. Księga jubileuszowa Profesora Herberta Szurgacza, Warszawa 2011, S. 500; E. Eichenhofer, Sozialrecht der Europäischen Union, Berlin 2006, S. 188.

3 Verordnung (EG) des Europäischen Parlaments und des Rates vom 29. April 2004 zur Koordinierung der Systeme der sozialen Sicherheit, Dz.U. UE. L.04.166.1.; nachstehend: Verordnung Nr. $883 / 2004$. 
Rechtssystemen der Mitgliedsstaaten vorgesehen wurden. Notwendig wird daher nicht nur eine Ermittlung des nach Vorschriften des zweiten Titels der Verordnung Nr. 883/2004 anwendbaren Rechts und die Anwendung des richtigen Vorrangsgrundsatzes bei Konkurrenz der Ansprüche auf Familienleistungen (Art. 68 und folgende der Verordnung). Es ist darüber hinaus auch eine auf allgemeine Grundsätze, Ziel und Koordinierungskern (Kumulationsverbot, Vorrangprinzip der Gesetzgebung eines einzigen Staates $^{4}$, Nichtverschlechterung der Rechtslage hinsichtlich der Berechtigung auf Leistungen aus der Sozialversicherung 5 , Koordinierung der Sozialversicherungssysteme und nicht nur lediglich eine Koordinierung im Rahmen von Leistungen, die durch die nationalen Rechtsordnungen zu einzelnen Zweigen der sozialen Sicherung hinzugerechnet werden) gestützte Entscheidungsfindung über folgende Problemfragen notwendig: Worauf beruht die Anspruchskonkurrenz bei Familienleistungen? In welchem Ausmaß bedarf deren Ermittlung einer Auswertung von Sozialversicherungssystemen der Mitgliedsstaaten und kommt es im konkreten Fall zum Zusammentreffen von Leistungen desselben Gegenstandes und Zieles? Es sollte nämlich darauf hingewiesen werden, dass „die Vorschriften der Verordnung eine materiell-rechtliche und nicht nur kollidierende und technisch-organisatorische Natur aufweisen, denn obwohl sie keine Akte sind, in denen die Voraussetzungen für die Entstehung der Versicherungspflicht geregelt werden, gestalten sie dennoch unmittelbar die materiell-rechtliche Stellung eines innerhalb des Unionsgebietes wandernden Arbeitnehmers und dessen Familie auf der Ebene der sozialen Sicherung". 6 Deshalb bereitet die Anwendung des obigen Konglomerates von Vorschriften und Regeln in der Praxis so viele Schwierigkeiten. Dies wird durch die Rechtsprechung der polnischen Verwaltungsgerichte belegt, die gleichzeitig die meist charakteristischen und problematischen Fälle im Hinblick auf Koordinierung der Familienleistungen im Verhältnis zwischen Polen und Deutschland schildert.

Ein anderer Grund für Probleme bei der Anwendung der Koordinierungsregeln von Familienleistungen in Polen ist oft fehlendes Wissen der Bürger darüber, dass es in der konkreten familiären Situation überhaupt zur Koordinierung kommt. Dies betrifft insbe-

4 Über Grundsätze der Koordinierung siehe Z. Brodecki (Hrsg.), Zatrudnienie i ochrona socjalna, Warszawa 2004, S. 384 ff.; L. Mitrus, Zakres (Fußn. 2), S. 492 ff.; A. Giżejewska/A. M. Światkowski, Zabezpieczenie Społeczne Komentarz, Kraków 2004, S. 45 ff.; G. Uścińska, Konsekwencje (Fußn. 2), S. 40.

5 H. Szurgacz, Prawo właściwe w przepisach o koordynacji systemów zabezpieczenia społecznego, in: Z. Niedbała (Hrsg.), Księga pamiątkowa w piątą rocznicę śmierci profesora Andrzeja Kijowskiego, Warszawa 2010, S. 291; A. M. Światkowski, Swoboda przemieszczania się w Europie a ochrona rodziny, Monitor Prawa Pracy 2007/6, S. 290; G. Uścińska, Nowe regulacje w zakresie koordynacji systemów zabezpieczenia społecznego, Praca i Zabezpieczenie Społeczne 2010/10, S. 2; dies., Koordynacja systemów zabezpieczenia społecznego. Aktualne problemy i wyzwania, in: $L$. Mitrus (Hrsg.), Studia z zakresu prawa pracy i polityki społecznej. Liber Amicorum prof. dr habil. Andrzej Marian Świątkowski, Kraków 2009, S. 543.

6 Urteil des Wojewódzki Sąd Administracyjny (WSA) in Gdańsk vom 19. Dezember 2007, II SA/Gd 609/07, LEX Nr. 342469; vgl. auch: T. Bińczycka-Majewska, Koordynacja systemów zabezpieczenia społecznego w Unii Europejskiej, Kraków 1999, S. 43. 
sondere zerfallene Familien, deren Angehörige keinen Kontakt miteinander unterhalten. In Bezug darauf ist die Aufnahme von staatlicher Informationstätigkeit sinnvoll, um sicherzustellen, dass die polnischen Staatsbürger, die das Freizügigkeitsrecht in Anspruch nehmen, über grundlegende Informationen betreffend die Rechtslage nach den Sozialversicherungssystemen der Mitgliedsstaaten und die Grundsätze der Koordinierung derselben verfügen. ${ }^{7}$

\section{B. Begriff und Koordinierung der Familienleistungen}

\section{Begriff der Familienleistungen nach polnischem Recht}

In der polnischen Rechtslehre versteht man unter dem Begriff der Familienleistungen eine Gesamtheit von dem Ziel nach zu definierenden Leistungen. Dieses Ziel ist die Familienunterstützung bei Ausführung ihrer Funktion und es soll durch die Zurverfügungstellung von Unterhaltsmitteln an die Familienangehörigen sowie durch Pflegeund Erziehungsmaßnahmen erreicht werden. ${ }^{8}$ Gleichzeitig wird ersichtlich, dass die so verstandene materielle Hilfe für die Familie in mehreren Rechtsakten und unter Anwendung von verschiedenen Methoden geregelt wird. Im weiteren Sinn werden zu den Familienleistungen auch Leistungen aus Unterhaltsfonds, Sozialrente, Hilfen für Pflegefamilien oder Familienrente gezählt ${ }^{9}$. Im engeren Sinn sind die Familienleistungen -

7 G. Uścińska, Konsekwencje (Fußn. 2), S. 42. Nebenbei kann zusätzlich bemerkt werden, dass seit dem Eintritt Polens in die Europäische Union und der Einbeziehung der Leistungen aus dem polnischen System der sozialen Sicherung in die Koordinierungsvorschriften 2009 die Anzahl der Personen, die Familienleistungen im Ausland beantragen, angestiegen ist. Beispielsweise sind von Mai 2004 bis Dezember 2005 bei den zuständigen polnischen Institutionen ca. 16000 Anträge polnischer Staatsbürger auf Familienleistungen im Ausland eingegangen. 2006 wuchs die Zahl auf bis zu 30931 Anträge ( . Adamska, Świadczenia rodzinne w Unii Europejskiej, Służba Pracownicz Nr. 7 2007, S. 23; G. Uścińska, Koordynacja (Fußn. 1), S. 48). Seit zwei Jahren sinkt diese Zahl. Während es 2009 noch 56400 solcher Fälle gab, hat sich die Zahl 2010 um 7100 gemindert. Dies ist ein Effekt der Wirtschaftskrise und der damit verbundenen kleineren Anzahl von Personen, die zur Arbeitssuche ins Ausland ziehen. Außerdem ist zu beachten, dass - wie aus den statistischen Daten über den Transfer der Familienleistungen folgt - seit Einbeziehung der polnischen Familienleistungen in das System der gemeinschaftlichen Koordinierung die meisten Anträge von Deutschland aus gestellt wurden (M. Drozdowicz, Koordynacja świadczeń rodzinnych, Polityka Społeczna 2008/1112, S. 43). Im Jahr 2010 wurden 21700 Anträge gestellt. Man kann annehmen, dass die völlige Öffnung des deutschen Arbeitsmarktes einen weiteren Anstieg der Anzahl von Polen, die Familienleistungen in Deutschland beantragen, zur Folge haben wird (M. Topolewska, Mniej rodzin ubiega się o zasiłek rodzinny na dziecko, Gazeta Prawna v. 05.05.2011 r.).

8 M. Lewandowicz-Machnikowska, in: R. Babińska-Górecka/dies. (Hrsg.), Świadczenia rodzinne Komentarz, Wrocław 2010, S. 42; vgl. W. Szubert, Ubezpieczenie społeczne. Zarys systemu, Warszawa 1987.

9 M. Lewandowicz-Machnikowska (Fußn. 8), S. 43; NSA-Entscheidung in Warszawa vom 4. März 2008, I OW 125/07, LEX Nr. 470976. 
laut Gesetz vom 28. November 2003 über die Familienleistungen ${ }^{10}$ - eine Unterstützungsform für Personen und Familien, die über niedriges Einkommen verfügen und dabei Kinder erziehen, oder wegen ihres Gesundheitszustands oder Alters einer zusätzlichen Unterstützung bedürfen ${ }^{11}$.

$\mathrm{Zu}$ den Familienleistungen gehört im Sinne der Vorschriften des polnischen Gesetzes über Familienleistungen Folgendes: Familienbeihilfe und Zulagen zur Familienbeihilfe, Pflegegeld und Pflegeleistung sowie einmalige Beihilfe bei Geburt, die kraft Ortsgesetzes ausgezahlt wird. ${ }^{12}$ Diese Leistungen weisen einen allgemeinen Charakter auf, werden aus dem Staatshaushalt finanziert und der Anspruch auf sie ist weder von einer vorherigen Versicherung noch von einem Anstellungsverhältnis abhängig.

Aus der Analyse der polnischen Rechtsprechung zur Koordinierung der oben dargestellten Leistungen folgt, dass sich die meisten Probleme im Rahmen der Anwendung von gemeinschaftlichen Vorschriften zwischen Polen und Deutschland auf Pflegeleistungen beziehen.

$\mathrm{Zu}$ Schwierigkeiten kommt es beispielsweise, wenn ein polnischer Bürger in Deutschland arbeitet und dessen behindertes Kind nebst der nicht erwerbstätigen Mutter in Polen wohnt. In diesem Sachverhalt besteht der Anspruch des Vaters auf Familienbeihilfe aus dem deutschen Rechtssystem zweifelsfrei. Das polnische Gesetz über Familienleistungen sieht jedoch außer Familienbeihilfe auch behinderungsbedingte Pflegeleistungen vor. Daher kann die Mutter zusätzlich Pflegeleistungen aus dem polnischen Rechtssystem der Familienleistungen beantragen. Dies folgt aus der Tatsache, dass die Pflegeleistungen im deutschen Recht auf der Ebene der Familienleistungen keine Äquivalente haben und zur Pflegeversicherung gehören, die nach dem Modell der gesetzlichen Krankenversicherung gestaltet wurde. Als solche stellen sie somit einen Teil der sozialen Versicherungen dar. ${ }^{13}$

In der polnischen Rechtsprechung wird einheitlich angenommen, dass die polnischen Pflegeleistungen für Kinder unter die Kategorie der Familienleistungen, wie sie in den Koordinierungsverordnungen definiert sind, fallen. ${ }^{14}$ Zur Begründung dieser Einstufung wird darauf hingewiesen, dass diese Leistungen von zuständigen Organen aufgrund rechtlich definierter Bedingungen ohne jegliche individuelle und subjektive Beurteilung gewährt werden, um teilweise die Ausgaben zu decken, die mit der für ein behindertes Kind zu leistenden Pflege und Hilfe durch andere Personen aufgrund ihrer Unfähigkeit zur selbständigen Existenz verbunden sind, also gerade zum Ausgleich von Unterhalts-

10 Dz.U. z 2006 r., Nr. 139, poz. 992 mit Änderungen.

11 Vgl. J. Jończyk, Prawo zabezpieczenia społecznego, Kraków 2001, S. 374; S. Nitecki, Procedura i tryb przyznawania świadczeń rodzinnych, Wrocław 2009, S. 17.

12 Art. 2 des Gesetzes.

13 J. Jończyk, Zagadnienia trwałej opieki pielęgnacyjnej, Praca i Zabezpieczenie Społeczne 2009/6, S. 3.

14 Urteile: WSA in Gdańsk vom 19. Dezember 2007, II SA/Gd 610/07; WSA in Gdańsk vom 19. Dezember 2007, II SA/Gd 609/07, LEX Nr. 342469; WSA in Białystok vom 21. August 2008, II SA/Bk 392/08, LEX Nr. 447871; WSA in Łódź vom 13. April 2010, II SA/Ld 185/10. 
kosten einer Familie, der ein behindertes Kind angehört. ${ }^{15}$ In den analysierten Entscheidungen wird außerdem auf das in der Begründung des polnischen Gesetzes über die Familienleistungen genannte Ziel dieser Leistungen hingewiesen. In dieser Begründung heißt es nämlich: „Das Ziel der Pflegeleistungen ist eine teilweise Deckung von Ausgaben, die durch die Familie in Bezug auf die Notwendigkeit einer Fürsorge und Pflege für ein behindertes Kind oder einen behinderten Erwachsenen getragen werden“".

\section{Anwendbarkeit bei grenzüberschreitenden Sachverhalten}

Gleichzeitig werden in der polnischen Rechtsprechung und im Schrifttum ${ }^{16}$ zwei verschiedene Meinungen hinsichtlich der Qualifizierung des Rechts auf Pflegeleistungen in dem Fall vertreten, in dem die nicht erwerbstätige Mutter eines behinderten Kindes in Polen wohnt und der Vater in einem anderen Mitgliedsstaat erwerbstätig und dort zum Bezug von Familienleistungen berechtigt ist.

Nach der ersten Ansicht kann der Anspruch auf Familienleistungen ausschließlich aus dem Rechtssystem eines einzigen Mitgliedsstaates erlangt werden. ${ }^{17}$

Unterstellt, dass in dem einleitend benannten Sachverhalt das deutsche Recht anzuwenden ist, wäre als Folge die Gewährung der im polnischen Gesetz über Familienleistungen vorgesehenen Pflegeleistungen zugunsten der Mutter eines behinderten Kindes nicht möglich. Dem Vater des Kindes stünden lediglich entsprechende Beihilfen und Familienleistungen aus dem deutschen System der sozialen Sicherung zu.

Demgegenüber wird in der Rechtsprechung und im Schriftum darauf hingewiesen, dass die Koordinierung der Familienleistungen ein Garant für die Durchsetzung des Freizügigkeitsprinzips und ein Hauptelement der Gleichbehandlungspolitik und Gewährung von sozialer Gerechtigkeit ist. ${ }^{18}$ Bei Festsetzung der Koordinierungsregeln für die nationalen Gesetzgebungen der Mitgliedsstaaten in den Koordinierungsverordnungen hat sich der Rat von dem fundamentalen Grundsatz des Vertragsrechts (schon im früheren EWG-Vertrag) inspirieren lassen, wonach das Unionsrecht den innerhalb der Union wandernden Arbeitnehmern eine Gesamtheit von in verschiedenen Mitgliedsstaaten

15 Urteile: WSA in Gdańsk vom 19. Dezember 2007, II SA/Gd 610/07; WSA in Gdańsk vom 19. Dezember 2007, II SA/Gd 609/07, LEX Nr. 342469; WSA in Białystok vom 21. August 2008, II SA/Bk 392/08, LEX Nr. 447871.

16 WSA-Urteil vom 17. März 2011, II SA/Op 685/10; vgl. WSA-Urteil in Rzeszów vom 24. Juli 2008, II SA/Rz 206/08; A. Wilk-Ilewicz/M. Drozdowicz/P. Gotębiowski, Glosa do wyroku WSA w Gdańsku z 28 listopada 2007 r., II SA/Gd 609/07, ZNSA 2010/2, S. 172 ff.

17 WSA-Urteil in Opole vom 17. März 2011, II SA/Op 685/10; vgl. WSA-Urteil in Rzeszów vom 24. Juli 2008, II SA/Rz 206/08, LEX Nr. 447875.

18 G. Uścińska, Koordynacja (Fußn. 1), S. 48; dies., Kierunki rozwoju świadczeń z zabezpieczenia społecznego na przykładzie wspólnotowej koordynacji, in: A. Patulski/K. Walczak (Hrsg.), Jedność w różnorodności. Studia z zakresu prawa pracy, zabezpieczenia społecznego i polityki społecznej. Księga pamiątkowa dedykowana Profesorowi Wojciechowi Muszalskiemu, Warszawa 2009, S. 33. 
erlangten Leistungen zu gewährleisten hat und dafür ein Günstigkeitsprinzip gilt. Das wird auch in den Präambeln der Koordinierungsverordnungen bestätigt. ${ }^{19}$ Somit darf die Anwendung der koordinierungsbezogenen Vorschriften in Bezug auf einen migrierenden Arbeitnehmer oder dessen Familienangehörige zu keinen negativen Folgen in Form einer Ablehnung des Rechts auf Familienleistungen oder einer Herabsetzung deren Höhe führen, falls diese Leistungen ausschließlich kraft nationaler Gesetzgebung zustehen sollten. Das Ziel der ex-Art. 48-51 EGV wäre nicht erreicht worden, wenn die Erwerbsmigration zu einer Einschränkung der Rechte hätte führen können, die dem Betroffenen bei Anwendung von ausschließlich nationaler Gesetzgebung zustanden. Die innerhalb der Union wandernden Personen dürfen sich in keiner schlechteren Lage befinden als die Personen, die ausschließlich von dem System der sozialen Sicherung eines Mitgliedsstaates erfasst werden. ${ }^{20}$

Gestützt auf die oben genannte Bedeutung und Rolle der Koordinierungsvorschriften wird in dieser Gruppe von verwaltungsgerichtlichen Entscheidungen angenommen, dass in dem Fall, dass die nicht erwerbstätige Mutter eines behinderten Kindes in Polen wohnt und der Kindesvater in einem anderen Mitgliedsstaat berufstätig und dort zu Familienleistungen berechtigt ist, weder das Prinzip des Vorrangs einer einzelnen maßgebenden Rechtsordnung noch die der Kumulationsvermeidung dienende und mit Aussetzung des Rechts auf kraft kollidierender Gesetzgebung zustehende Familienleistungen verbundene Vorrangregel anzuwenden sei.

In der vorgenannten Rechtsprechung wird auch geprüft, ob die jeweilige, in dem System der Familienleistungen in Polen enthaltene Pflegeleistung in dem Familienleistungssystem eines anderen Mitgliedsstaates ihr Äquivalent findet. ${ }^{21}$ Es wird erwähnt, dass für den Fall, dass die Rechtsordnung des Erwerbsstaates die jeweilige Leistungsart nicht vorsieht und das Recht auf diese Leistung der Partei kraft der im Wohnstaat geltenden Vorschriften zusteht (kraft polnischen Gesetzes über die Familienleistungen), es dann an Gründen fehlt, um die Leistung auszusetzen. ${ }^{22}$ Keine von den in den Koordinierungsverordnungen enthaltenen Vorschriften sieht nämlich eine solche Sanktion vor.

19 Pkt. 1 der Präambel der Verordnung Nr. 833/2004.

20 Urteile: WSA in Gdańsk vom 19. Dezember 2007, II SA/Gd 610/07; WSA in Gdańsk vom 19. Dezember 2007, II SA/Gd 609/07, LEX Nr. 342469; diese Auffassung hat der Gerichtshof u.a. auch in der Entscheidung von 1977 in der Rechtssache Strhel angenommen (Urteil in der Rechtssache Strhel c. Nationaal Pensioenfounds voor Monwerkers / Belgia, Zbiór orzeczeń ETS z 1977 roku, S. 211); T. Binczycka-Majewska, Koordynacja (Fußn. 6), S. 43; Vorabentscheidung des EuGH, Rs. C24/75 (Petroni/ONTPS), Slg. 1975 I-1149; Komentarz do rozporządzenia Rady (EWG) Nr. 1408/71 z dnia 14 czerwca 1971 r. w sprawie stosowania systemów zabezpieczenia społecznego do pracowników, osób prowadzących działalność na własny rachunek i do członków ich rodzin przemieszczających się we Wspólnocie, G. Uścińska (Hrsg.), Ministerstwo Polityki Społecznej, Warszawa 2005, S. $314 \mathrm{ff}$.

21 WSA-Urteil in Białystok vom 21. August 2008, II SA/Bk 392/08, LEX Nr. 447871. WSA-Urteil in Gdańsk vom 19. Dezember 2007, II SA/Gd 610/07, LEX Nr. 347925. 


\section{Zur Frage der Kumulierung}

Im Lichte der oben genannten Auffassungen erhielte der in Deutschland arbeitende Vater im oben genannten Ausgangssachverhalt entsprechende Beihilfen und Familienleistungen aus dem deutschen System der Familienleistungen und die anderen, in Polen wohnhaften Familienmitglieder (das behinderte Kind und die nicht erwerbstätige Mutter) würden ein Recht auf die durch das polnische Gesetz über Familienleistungen geregelten Pflegeleistungen erwerben.

Gleichzeitig ist zu bemerken, dass der in Deutschland arbeitende Vater grundsätzlich dem deutschen Sozialversicherungssystem untersteht, darunter auch der Pflegeversicherung, aus welcher er oder die Angehörigen seiner Familie ein Recht auf die durch die Behinderung des Kindes bedingten Leistungen erwerben können. ${ }^{23}$

Dies bedeutet, dass der Vater eines in Polen wohnhaften behinderten Kindes, dem aus dem polnischen Familienleistungssystem Pflegeleistungen zustehen, im Zusammenhang mit der in Deutschland ausgeübten Erwerbstätigkeit dort entsprechende Pflegeleistungen aus der Pflegeversicherung beantragen könnte.

Somit entsteht die Frage, ob es in dem genannten Sachverhalt möglicherweise nicht zu einem Zusammentreffen von Leistungen im Sinne des Art. 10 der Verordnung Nr. 883/2004 (Art. 12 der Verordnung Nr. 1408/71) kommt. Nach der obigen Vorschrift gewährt und erhält die Verordnung Nr. 883/2004 keinen Anspruch auf mehrere gleichartige Leistungen aus derselben Versicherungszeit aufrecht. Dieser Grundsatz bezieht sich auf die Sozialversicherungssysteme der Mitgliedsstaaten, die eine aus verschiedenen Leistungen bestehende Gesamtheit darstellen. Diese Leistungen werden in den einzelnen Gesetzgebungen anders qualifiziert, deren gemeinsames Kriterium ist dabei aber die Kategorie des sozialen Risikos. Wie nämlich im Schrifttum festgestellt wird, wirkt die genannte Vorschrift solchen Situationen entgegen, in denen eine Person zum Empfänger mehrerer gleichartiger Leistungen für den Fall desselben Risikos würde. ${ }^{24}$ Die Verordnungsvorschriften sollen also zweckmäßigerweise u.a. einer Konkurrenz von analogen Leistungen, die in mehreren Systemen der Mitgliedsstaaten vorgesehen wurden, entgegenwirken. ${ }^{25}$ Der Europäische Gerichtshof hat bei Qualifizierung einer konkreten Leistung im Sozialversicherungssystem mit Rücksichtnahme auf deren rechtlichen Charakter, Ziel und Funktion mehrfach angenommen, dass die konkrete Leistung mit einem anderen als dem in der nationalen Gesetzgebung angenommenen Risiko verbunden sei. ${ }^{26}$ Bei Ermittlung von Leistungsgruppen derselben Art kann man sich also

23 Art. 21 der Verordnung Nr. 833/2004; A. Wilk-Ilewicz/M. Drozdowicz/P. Golębiowski, Glosa do wyroku (Fußn. 16), S. 173.

24 A. Giżejewska/A.M. Świqtkowski, Zabezpieczenie społeczne (Fußn. 4), S. 45.

25 Vgl. H. Szurgacz, Prawo (Fußn. 5), S. 292.

26 In Bezug auf das Altersrisiko in den Entscheidungen EuGH-Urteil vom 02. Mai 1996, C-206/94 (Paleetta IL), Slg. 1996, I-2357; vom 10. Januar 1980, C-69/79 (Jordens-Vosters), Slg. 1980, I-75; vom 05. März 1998, C-160-96 (Molenaar), Slg. 1998, I-843; vom 24. September 1998, C-132/96 
nicht ausschließlich an formalen Kriterien orientieren, d.h. nach der Zugehörigkeit einer Leistung zum jeweiligen Zweig der sozialen Sicherung in der nationalen Rechtsordnung oder in Bezug auf die Struktur des Sozialsicherungssystems, sondern nach dem sachlichen Regelungsbereich, dem Ziel, den Erwerbsvoraussetzungen und der Empfängerkategorie. ${ }^{27}$

Das soziale Risiko ist also in erster Linie ein Kriterium, das die Feststellung zulässt, ob im konkreten Fall ein Zusammentreffen von Leistungen vorkommt und es entscheidet folglich über die Wahl der einschlägigen Anti-Kumulationsregel. Das heißt, dass man sich bei Beachtung des Anti-Kumulationsprinzips nicht lediglich auf einen Vergleich der Leistungen beschränken kann, die in den kollidierenden Rechtsordnungen der Mitgliedsstaaten demselben Zweig der sozialen Sicherung zugeordnet wurden. ${ }^{28}$ Es sollte darüber hinaus ermittelt werden, ob das gesamte System der sozialen Sicherung des jeweiligen Mitgliedsstaates eine Leistung vorsieht, die der im System eines anderen Mitgliedsstaates vorhandenen Leistung entspricht. ${ }^{29}$ An dieser Stelle ist zu erwähnen, dass das Anti-Kumulationsprinzip in den Vorschriften des polnischen Gesetzes über Familienleistungen identisch verstanden wird - diese Vorschriften verweisen bei einem Zusammentreffen von Leistungen auf solche Kriterien wie Ziel, sachlicher Regelungsbereich, Erwerbsvoraussetzungen und Empfängerkreis. ${ }^{30}$

Ein Vergleich der in kollidierenden Gesetzgebungen vorkommenden Leistungen nach dem Kriterium des sozialen Risikos (Ziel, Erwerbsvoraussetzungen, Empfängerkategorie, sachlicher Regelungsbereich) kann gegebenenfalls zur Feststellung eines Zusammentreffens von Leistungen führen, die in den nationalen Rechtsordnungen ver-

(Stinco, Panfilo), Slg. 1998, I-5225; vom 11. Juni 1998, C-297/96 (Partridge), Slg. 1998, I-3467; weiter dazu: G. Uścińska/B. Kazenas (Hrsg.), Orzeczenia Europejskiego Trybunału Sprawiedliwości w dziedzinie zabezpieczenia społecznego, wersja szeroka i wersja skrócona, Warszawa 2002.

27 Ähnlich: A. Giżejewska/A.M. Świq̨tkowski, Zabezpieczenie społeczne (Fußn. 4), S. 46 und das dort angeführte EuGH-Urteil Vander Burt-Craig 238/81 [1983] ECR 1385; vgl. EuGH-Urteil vom 11. September 1995, Rs. C-98/94 (Schmidt), in: G. Uścińska/B. Kazenas (Hrsg.), Orzeczenia (Fußn. 26), S. 222 ff.; Vorabentscheidung des EuGH vom 16. Juli 1992, Rs. C-78/91 (Rose Hughes), Slg. 1992, I-4839.

28 So: WSA-Urteil in Białystok vom 21. August 2008, II SA/Bk 392/08, LEX Nr. 447871.

29 WSA-Urteil in Łódź vom 13. März 2010, II SA/Łd 185/10; WSA-Urteil in Gdańsk vom 19. Dezember 2007, II SA/Gd 610/07, LEX Nr. 347925; WSA-Urteil in Gdańsk vom 28. November 2007, II SA/Gd 609/07.

30 Z.B. Art. 16 Abs. 5a des Gesetzes, der bestimmt, dass kein Anspruch auf die Pflegeleistung besteht, wenn dem Familienmitglied im Ausland eine Leistung zur Deckung der mit der Pflege über diese Person verbundenen Kosten zusteht, es sei denn, die Vorschriften über die Koordinierung der Systeme der sozialen Sicherung oder zweiseitige Verträge über Sozialsicherung bestimmen Abweichendes; Art. 17 Abs. 5 Pkt. 5 des Gesetzes, wonach kein Anspruch auf die Pflegeleistung für eine pflegebedürftige Person besteht, wenn das Familienmitglied im Ausland zu einer Leistung zur Deckung der mit der Pflege über diese Person verbundenen Kosten berechtigt ist, es sei denn, die Vorschriften über die Koordinierung der Systeme der sozialen Sicherung oder zweiseitige Verträge über Sozialsicherung bestimmen Abweichendes. 
schiedenen Zweigen der sozialen Sicherung zugeordnet werden (z.B. Familienleistungen und Leistungen aus der Versicherung im Krankheitsfall). Es entsteht dann ein Problem bezüglich der Auswahl richtiger Anti-Kumulationsregeln, die in den Koordinierungsverordnungen für jeden dort benannten Zweig der sozialen Sicherung explizit bestimmt wurden.

Das obige Problem wird durch die Feststellung gelöst, dass die in der Verordnung Nr. 883/2004 sowie in der Rechtsprechung der EuGH enthaltenen Begriffe für die Zuordnung einzelner Leistungen innerhalb von Zweigen der sozialen Sicherung von entscheidender Bedeutung sind.

\section{Begriff der Familienleistungen nach Unionsrecht}

Die Vorschriften der Koordinierungsverordnungen enthalten einen allgemeinen Begriff der Familienleistungen. Dies heißt, dass über den Bereich und den Begriff der Familienleistungen, die hinsichtlich der Koordinierung den in den Vorschriften des 8. Kapitels der Verordnung Nr. 883/2004 bestimmten Grundsätzen unterliegen, nicht die erwähnte Leistungseinstufung gemäß dem nationalen Recht der Mitgliedsstaaten, sondern die angesprochenen Verordnungsregelungen zu entscheiden haben.

Laut Art. 1 Buchstabe z der Verordnung Nr. 883/2004 umfasst der Begriff „Familienleistung“" alle Sach- oder Geldleistungen, die den Familienausgaben zu entsprechen haben, jedoch unter Ausschluss von Unterhaltsvorschüssen sowie besonderen Geburtsund Adoptionsbeihilfen, die im Anhang I erwähnt wurden. Es kann also angenommen werden, dass der Schutzgegenstand bei Familienleistungen die Familienlasten sind. ${ }^{31}$ Aus weiteren Verordnungsvorschriften, darunter insbesondere aus der Vorschrift des Art. 68, folgt, dass es sich dennoch um ein engeres Verständnis dieses Begriffs handelt - und somit um die mit Kindesunterhalt und Kindererziehung sowie mit Obhut über die Kinder verbundenen Kosten. Als Kriterium zur Feststellung des Rechtsvorrangs eines Staates (sog. Anknüpfungsgegenstand) dient jeweils der Kinderwohnort und nicht der Wohnort anderer Familienangehöriger. Außerdem soll der Begriff „Familienausgaben” gemäß der Rechtsprechung des Europäischen Gerichtshofes in Bezug auf die Beteiligung öffentlicher Ausgaben in Haushaltsbudgets zur Räumung finanzieller, mit Kindesunterhaltskosten verbundener Barrieren interpretiert werden. 32

31 G. Uścińska, Kierunki rozwoju (Fußn. 18), S. 333.

32 EuGH-Urteil vom 15. März 2001, Rs. C-85/99 (Offermanns), Slg. 2001, I-2261, in: G. Uścińska:, Orzecznictwo, S. 144; ähnlich sieht den Charakter von Familienleistungen A. Giżejowska/A. M. Światkowski, Zabezpieczenie Społeczne Komentarz, Kraków 2004, S. 155 f.; EuGH-Urteil vom 7. November 2002, Rs. C-333/00 (Maaheimo), Slg. 2002, I-10087; vom 20. Januar 2005, Rs. C-302/02 (Nils Laurina Effing), Slg. 2005, I-553 unter Verweis auf seine vorherigen Entscheidungen vom 15. März 2001, Rs. C-85/99 (Offermans), Slg. 2001, I-2261 sowie vom 5. Februar 2002, Rs C-255/99 (Humer), Slg. 2002, I-1205. 
Abgesehen davon, dass die Verordnung Nr. 883/2004 auf eine ausdrückliche Aufteilung der Leistungen in Familienbeihilfen und Familienleistungen im Gegensatz zur Verordnung Nr. 1407/81 verzichtet, weist sie dennoch im Pkt. 34 der Präambel darauf hin, dass die Familienleistungen einen sehr breiten Umfang aufweisen, indem sie Schutz in Situationen bieten, die als klassisch bezeichnet werden könnten, aber auch in anderen Fällen von besonderem Charakter, wobei die letzte Leistungsart in den verbundenen Rechtssachen C-245/94 und C-312/94 (Hoeveri Zachov) sowie in der Rechtssache C275/96 (Kuusijärvi) zum Urteilsgegenstand des Europäischen Gerichtshofes geworden ist. Klassische Familienleistungen sind Familienbeihilfen, die sich auf allgemein gefasste Familienunterstützung bei Deckung von Kindesunterhalts- und Erziehungskosten beziehen, jedoch ohne die Quellen dieser Kosten genau zu bestimmen, und stellen periodische, lediglich je nach Anzahl und Alter der Familienangehörigen zu gewährende Leistungen dar. Im Schrifttum wird betont, dass der Anspruch auf Familienleistungen dem Grunde nach von der fehlenden Möglichkeit selbstständiger Unterhaltsbestreitung der Personen abhängt, denen diese Leistungen zustehen. ${ }^{33}$ Diese Personen können wegen ihres Alters, einer Ausbildungsfortsetzung oder einer eingeschränkten Körperfunktionsfähigkeit keine Berufstätigkeit aufnehmen. ${ }^{34}$ Unter Berücksichtigung der in Pkt. 34 der Präambel zur Verordnung Nr. 883/2004 angeführten EuGH-Rechtsprechung sind als Familienleistungen besonderen Charakters solche Leistungen anzusehen, die sich auf konkrete, mit Kindesunterhalt und Kindererziehung verbundene Bedürfnisse beziehen. Sie gleichen z.B. die Ausgaben für Schulausbildung eines Kindes aus.

\section{Folgerungen für die Qualifizierung nach polnischem Recht}

An dieser Stelle soll ferner angedeutet werden, dass in der polnischen Rechtsprechung ein gewisses Einstufungsproblem von polnischen Pflegeleistungen in der Kategorie der Familienleistungen ersichtlich wird. Es wird nämlich eingesehen, dass das Pflegegeld für ein Kind den Charakter einer Familienleistung aufweisen könne und das gleiche Pflegegeld in Bezug auf Erwachsene unter Umständen als eine Leistung im Krankheitsfall anzusehen wäre. ${ }^{35}$

Wie es scheint, soll das sachliche Kriterium, also die Frage nach dem Leistungsempfänger, nicht als alleinige Voraussetzung über die Zugehörigkeit einer Leistung zum jeweiligen Zweig der sozialen Sicherung entscheiden. Eine Auswertung in diesem Rahmen soll komplex verlaufen, unter Berücksichtigung des Zieles, der Erwerbsvoraussetzungen sowie des sachlichen Regelungsbereiches der jeweiligen Leistung.

Laut den polnischen Gesetzen über Familienleistungen steht die Pflegeleistung einer unterhaltspflichtigen Person zu, die einen Dauer- oder Langzeitpflegebedürftigen be-

33 Vgl. A. Giżejowska/A. M. Światkowski, Zabezpieczenie (Fußn. 32), S. 176.

34 A. Giżejowska/A. M. Światkowski, Zabezpieczenie (Fußn. 32), S. 176.

35 WSA-Urteil in Opole vom 17. März 2011, II SA/Op 685/10. 
treut, während das Pflegegeld derjenigen Person zusteht, die einer solchen Pflege bedarf. Hiermit sollen die mit der Pflege verbundenen Kosten gedeckt werden. Die Zielpersonen dieser Leistungen sind nicht nur ausschließlich Kinder und deren Eltern. In mehreren Fällen stehen diese Leistungen Personen im fortgeschrittenen Alter zu, z.B. steht das Pflegegeld außer behinderten Kindern auch jeder Person ab dem vollendeten 75. Lebensjahr zu. Die Pflegeleistung kann dem Betroffenen genauso wie bei der Kinderpflege auch bei der Pflege eines anderen Familienangehörigen zustehen, wie z.B. Großvater, Schwester, Elternteil, Ehegatten. ${ }^{36}$

Aus dem Gesagten folgt, dass das Ziel der polnischen Familienleistungen die Sicherstellung einer ordnungsgemäßen Eingliederung von Behinderten oder Personen im fortgeschrittenen Alter in die Gesellschaft ist, u.a. durch Gewährung finanzieller Leistungen zur Kostendeckung einer ,,in Eigenregie" organisierten Pflege, darunter auch Pflege durch Verwandte. ${ }^{37}$ Diese Leistungen gleichen die Kindesunterhaltskosten nicht aus, sondern stellen bestimmte Finanzmittel für die Rehabilitation von Behinderten oder deren Pflege sicher. Somit entsprechen sie nicht dem in der Verordnung Nr. 883/2004 definierten Begriff von Familienleistungen.

36 Das Pflegegeld wird zur teilweisen Kostendeckung der im Zusammenhang mit einer wegen Unfähigkeit zur selbstständigen Existenz benötigten Pflege und Hilfe Dritter gewährt. Diese Leistung steht einem behinderten Kind, Behinderten im Alter von über 16 Jahren bei Befund einer Schwerbehinderung sowie Personen ab dem vollendetem 75. Lebensjahr zu. Das Pflegegeld steht außerdem auch Behinderten über 16 Jahren zu, wenn ein Befund über einen gemäßigten Behinderungsgrad vorliegt und diese Behinderung bis zur Vollendung des 21. Lebensjahres entstanden ist. Das Pflegegeld wird unabhängig vom Einkommen gewährt und beträgt 153 PLN monatlich (Art. 16 des Gesetzes i.V.m $\S 1$ Pkt. 12 der Verordnung des Ministerrates vom 11. August 2009 w sprawie wysokości dochodu rodziny albo dochodu osoby uczącej się stanowiących podstawę ubiegania się o zasiłek rodzinny oraz wysokości świadczeń rodzinnych [über die Höhe des Familieneinkommens oder Einkommens von Personen in Ausbildung zur Festsetzung der Bemessungsgrundlage bei Beantragung der Familienbeihilfe und Familienleistungen ] (Dz.U. Nr. 129, Pos. 1058). Die Pflegeleistung wird hingegen im Zusammenhang mit dem Verzicht auf Anstellung oder einer anderen Erwerbstätigkeit der Mutter oder dem Vater sowie anderen Personen, die laut Vorschriften des Gesetzes vom 25. Februar 1964 - Kodeks rodzinny i opiekuńczy [das Familiengesetzbuch] (Dz.U. Nr 9, poz. 59, mit Änderungen) unterhaltspflichtig sind, sowie auch dem faktischen Kinderbetreuer gewährt - wenn sie keine Erwerbstätigkeit aufnehmen oder auf diese verzichten, um eine für behindert erklärte Person zu pflegen, und folgende Voraussetzungen vorliegen: Notwendigkeit von Dauer- oder Langzeitpflege oder Hilfe Dritter im Zusammenhang mit einer erheblich eingeschränkten Fähigkeit zur selbstständigen Existenz und der Notwendigkeit einer dauerhaften, täglichen Anteilnahme des Kinderbetreuers an dessen Behandlung, Rehabilitation und Ausbildung oder Pflege eines Schwerbehinderten. Darüber hinaus kann die Pflegeleistung auch einer anderen als im ersten Grad verwandten, unterhaltspflichtigen Person zustehen und zwar in dem Fall, wenn es keine Verwandten ersten Grades gibt, oder wenn die Verwandten ersten Grades die Pflege nicht aufnehmen können. Die Pflegeleistung wird unabhängig vom Einkommen gewährt und beträgt 520 PLN monatlich (Art. 17 des Gesetzes).

37 J. Knyżewska/G. Sypniewska, Europejskie systemy zabezpieczenia społecznego. Niemcy, Sł. Pracownicza, 2008/1, S. 32. 
Die obige Schlussfolgerung soll gleichzeitig durch die Feststellung ergänzt werden, dass - unter Berücksichtigung der Rechtsprechung des EuGH ${ }^{38}$ - die polnischen Pflegeleistungen nicht aus der Koordinierung ausgeschlossen werden können. Das Pflegegeld und die Pflegeleistung sind automatisch zu gewährende Leistungen für Personen, die bestimmte objektive, von individuellen Bedürfnissen unabhängige Kriterien erfüllen. Außerdem nimmt das Gesetz an, dass die benannten Leistungen im Koordinierungsfall nicht zustehen. ${ }^{39}$ Diese Vorschriften sollen einer Kumulation von nationalen und ausländischen Leistungen, deren Erwerbsvoraussetzung Pflege einer pflege- oder hilfsbedürftigen Person ist, sowie einer Kumulation von Leistungen, die zur Deckung von Ausgaben wegen der Pflege einer behinderten Person gewährt werden, entgegenwirken. Diese Leistungen dürfen also - in Sachverhalten mit Auslandsbezug - nicht lediglich in Anlehnung an die polnische Gesetzgebung gewährt und ausgezahlt werden.

Der EuGH hat festgestellt, dass das Ziel der Leistungen, die eine Verbesserung des Gesundheitszustandes und der Lebensbedingungen pflegebedürftiger Personen verfolgen, grundsätzlich eine Ergänzung von Leistungen aus der Krankenversicherung oder aus einem anderen Schutz im Krankheitsfall gewährenden System ist, und aus diesem Grund als Leistungen im Krankheitsfall im Sinne der Vorschriften der Koordinierungsverordnungen verstanden werden können. 40

Trotz der Zweifel, die mit der Koordinierung von Leistungen bei Langzeitpflege verbunden waren, insbesondere der Zweifel an deren Zugehörigkeit zu dem im Koordinierungsverfahren betroffenen sozialen Risiko, ist in die Verordnung Nr. 833/2004 trotz Modifizierung des sachlichen Geltungsbereiches keine Koordinierung der Leistungen bei Langzeitpflege aufgenommen worden. Das Risiko der Hilfebedürftigkeit ist nicht als eigenständiges Risiko qualifiziert worden. ${ }^{41}$ Zugleich rechnet die Verordnung $\mathrm{Nr}$. 883/2004 die Leistungen bei Langzeitpflege ausdrücklich dem Zweig von krankheitsbedingten Leistungen zu. ${ }^{42}$ Wie im Schrifttum betont wird, weisen die Leistungen bei Langzeitpflege in einigen Ländern der Welt einen beitragsfreien Charakter auf. ${ }^{43}$ Nichtsdestotrotz erlangt die Finanzierungsmethode derartiger Leistungen keine entscheidende Rolle bei deren Einstufung, darunter bei der Feststellung, ob sie zu Familienleistungen oder zu einem anderen, auf Versicherung und Beiträge gestützten Zweig der sozialen Sicherung angehören. Das Koordinierungsziel ist - wie bereits angedeutet der Schutz von migrierenden Arbeitnehmern und deren Familienangehörigen und nicht die Schaffung eines supranationalen Sozialversicherungssystems oder die Beseitigung

38 Vgl. EuGH-Urteil vom 16. Juli 1992, Rs. C-78/91 (Hughes), Slg. 1992, I-4839; vom 10. Oktober 1996, Rs. C-312/94 (Hoever Zachow), Slg. 1996, I-4895; WSA-Urteil in Gdańsk vom 19. Dezember 2007, IISA/Gd 609/07, Lex 342469.

39 Art. 16 Abs. 5 a sowie Art. 17 Abs. 5 Pkt. 5 des Gesetzes.

40 Long Term Care-LTC; EuGH-Urteil vom 08. März 2001, Rs. C-215/99 (Jauch), Slg. 2001, I-1901; vom 21. Februar 2006, Rs. C-286/03 (Hose), Slg. 2006, I-1771.

41 G. Uścińska, Nowe (Fußn. 5), S. 6 f.

42 Titel III, Kapitel I der Verordnung Nr. 833/2004.

43 G. Uścińska, Nowe (Fußn. 5), S. 6 f. 
von Differenzen zwischen nationalen Systemen. ${ }^{44}$ Die Koordinierung gilt nämlich unabhängig von Charakter und Finanzierungsquelle der vom Regelungsbereich der Koordinierungsvorschriften umfassten Leistungen in den jeweiligen Mitgliedsstaaten. ${ }^{45}$

Trotz formaler Zugehörigkeit des Pflegegeldes und der Pflegeleistung zu den Familienleistungen im Lichte der polnischen Rechtsordnung sind diese Leistungen gemeinschaftsrechtlich zu den Leistungen im Krankheitsfall zu zählen; der Anspruch auf sie ist folglich unter Berücksichtigung der Vorschriften des Kapitels I der Verordnung (Art. 17-22) und nicht der Koordinierungsregeln des 8. Kapitels zu bewerten. Eine abweichende Auffassung könnte zum Verstoß gegen das Leistungskumulationsverbot führen. So ist auch der Sachverhalt zu bewerten, in dem der in Deutschland arbeitende Vater entsprechende Beihilfen und Familienleistungen aus dem deutschem Familienleistungssystem erhält und in Deutschland entsprechende Pflegeleistungen aus der Pflegeversicherung erwerben kann, und die anderen in Polen wohnhaften Familienangehörigen (das behinderte Kind und die nicht erwerbstätige Mutter) die in dem polnischen Gesetz über die Familienleistungen vorgesehenen Pflegeleistungen beziehen. Eine Einordnung der polnischen Pflegeleistungen in die Kategorie der Leistungen aus Versicherung im Krankheitsfall (Langzeitpflege) ermöglicht es, die Kumulation zu vermeiden unter Anwendung der Regeln des I. Kapitels der Verordnung (Art. 17-22).

\section{Weitere Problemstellungen}

Die übrigen mit der Koordinierung von Familienleistungen verbundenen Probleme, auch etwa im Verhältnis zwischen Polen und Deutschland, entstehen nicht aus Schwierigkeiten bei der Interpretation der Verordnungsvorschriften, sondern beziehen sich auf die Auslegung und Anwendung folgender, im polnischen Gesetz über Familienleistungen geregelter Institution: die Aufhebung eines das Recht auf Leistungen gewährenden Beschlusses im Zusammenhang mit der Leistungskoordinierung sowie ungerechtfertigt erhaltenen Leistungen.

Das betrifft die Sachlage von zerfallenen Familien, deren Angehörige keinen Kontakt miteinander unterhalten. Zum Beispiel, wenn ein Kindesvater die in Polen wohnhafte, nicht erwerbstätige Mutter des Kindes nicht über die Aufnahme einer Beschäftigung in Deutschland informiert. Sollte die Mutter in einem solchen Fall einen Beschluss über die Gewährung von Familienleistungen erlangen, ist dann ein derartiger Beschluss (des Gemeindevorstehenden, Bürgermeisters oder Stadtpräsidenten) gemäß Vorschriften des polnischen Gesetzes über Familienleistungen wegen des Erlasses durch ein unzuständi-

44 G. Uścińska, Koordynacja (Fußn. 1), S. 44.

45 Vgl. J. Boruta, Swoboda przepływu osób, in: J. Barcz (Hrsg.), Prawo Unii Europejskiej, Prawo materialne i polityki, Warszawa 2003, S. 71; vgl. auch A. Giżejowska/A. M. Świqtkowski, Zabezpieczenie (Fußn. 32), S. 155. 
ges Organ aufzuheben. ${ }^{46}$ Zur Aufhebung dieses Beschlusses ist dann alleine die Feststellung ausreichend, dass der Vater der deutschen Rechtsordnung untersteht, und zwar unabhängig von einem Antrag auf diese Leistung und ohne Rücksicht auf den das Recht gewährenden Beschluss. ${ }^{47}$ In dieser Situation entscheidet ja das Organ nicht über den Anspruch einer Person auf Familienleistungen, sondern über die Zuständigkeit des Organes zum Erlass einer Entscheidung bezüglich dieser Leistungen. Eine Leistungsgewährung oder -ablehnung erfolgt im Wege einer gesonderten Entscheidung des Organes, das für die mit der Koordinierung von Familienleistungen verbundenen Angelegenheiten zuständig ist (Woiwodschaftsmarschall).

Die Notwendigkeit der Anwendung der Koordinierungsregeln offenbart sich oftmals erst nach mehreren Bezugsjahren von Familienleistungen durch die Mutter in Polen. Vor dem Hintergrund des obigen Sachverhaltes wird häufig festgestellt, dass Polen nicht der für die Auszahlung von Familienleistungen zuständige Staat ist. Gemäß den polnischen Gesetzen entsteht dann das Problem der Qualifizierung der in Polen ausgezahlten Leistungen als ungerechtfertigt erhaltene Leistungen. Gemäß dieser Vorschriften werden diejenigen Leistungen als ungerechtfertigt erhaltene Familienleistungen bezeichnet, die für den Zeitraum ab dem Tag, an dem die Person zu Familienleistungen in einem anderen Mitgliedsstaat im Zusammenhang mit der Anwendung von Vorschriften über die Koordinierung der Sozialversicherungssysteme berechtigt war, bis zum Tag des Beschlusserlasses über Aufhebung des die Familienleistungen gewährenden Beschlusses hin ausgezahlt worden sind 48 .

Bei der Auslegung der obigen Regelung lassen sich in der polnischen Rechtsprechung und im Schrifttum drei verschiedene Meinungen ausmachen. Nach der ersten sei für die Feststellung eines ungerechtfertigten Charakters der bezogenen Leistungen im Hinblick auf den zu beurteilenden Sachverhalt ausreichend, dass der Kindesvater ein Arbeitsverhältnis in Deutschland eingegangen ist. Dieser Umstand entscheide automatisch über die Qualifizierung der in Polen ausgezahlten Leistungen als ungerechtfertigt erhaltene Leistungen. ${ }^{49}$ Die zweite Ansicht nimmt an, dass der Gesetzgeber die Kategorisierung der an die Kindesmutter ausbezahlten Familienleistung als ungerechtfertigt bezogene Leistung nicht von dem Umstand einer Antragstellung auf diese Leistungen durch den Kindesvater in Deutschland abhängig gemacht hat, sondern alleine vom Erwerb eines Anspruchs auf diese Leistungen durch ihn. ${ }^{50}$ Anspruchsbegründend wirkt dabei die Erfüllung derjenigen Voraussetzungen, die für einen Anspruchserwerb auf eine gleichartige Leistung nach deutschem Recht nötig wären, und nicht die Antragstellung oder Leistungsgewährung in Form der Auszahlung. Gemäß der dritten Auffassung sollen hingegen als ungerechtfertigt erhaltene Leistungen ausschließlich die Familien-

46 Art. 23a Abs. 5 des Gesetzes.

47 R. Babińska-Górecka, in: dies./M. Lewandowicz-Machnikowska, Świadczenia (Fußn. 8), S. 285.

48 Art. 30 Abs. 2 Pkt. 4 des Gesetzes.

49 SKO in Wrocław.

50 WSA-Urteil in Kielce, II SA/KE 45/09, http://orzeczenia.nsa.gov.pl. 
leistungen angesehen werden, die dem Kindesvater in Deutschland und der Kindesmutter in Polen für dieselben Versicherungszeiten ausbezahlt worden sind. ${ }^{51}$

Unter Berücksichtigung des Kernes und der Grundsätze der Koordinierung sowie des Wortlautes der oben zitierten Gesetzesvorschrift des Art. 30 Abs. 2 Pkt. 4 ist abschließend zu folgern, dass die Einstufung der Leistungen als durch die Mutter ungerechtfertigt bezogen aufgrund der polnischen Vorschriften nur unter der Bedingung erfolgen kann, dass der Kindesvater in Deutschland wohnt und die Voraussetzungen für den Anspruchserwerb auf die im deutschen Recht vorgesehenen Familienleistungen erfüllt.

51 S. Nitecki, Świadczenia (Fußn. 11), S. 180. 
